\title{
Increasing prevalence of type 2 diabetes in a Scottish population: effect of increasing incidence or decreasing mortality?
}

\author{
J. M. M. Evans • K. N. Barnett • S. A. Ogston • \\ A. D. Morris
}

Received: 18 September 2006 / Accepted: 8 December 2006 / Published online: 16 January 2007

(C) Springer-Verlag 2007

\begin{abstract}
Aims/hypothesis We examined incidence, prevalence and mortality from type 2 diabetes mellitus in a Scottish population over 12 years, and evaluated the effects on prevalence of increasing incidence and decreasing mortality. Materials and methods We used a diabetes clinical information system in Tayside (population 387,908), Scotland, to identify new cases of type 2 diabetes between 1993 and 2004 and to calculate incidence rates and mid-year prevalence. We defined mortality rates as the number of deaths of diabetic people divided by mid-year prevalence. We used logistic and Poisson regression to analyse trends. We then modelled the increase in prevalence for each year for three scenarios, based on whether mortality or incidence rates remained unchanged from 1993.

Results There was a doubling in incidence and prevalence of type 2 diabetes in Tayside over the 12 years, with statistically significant increasing trends of 6.3 and $6.7 \%$ per year respectively. The mortality rate decreased. If
\end{abstract}

J. M. M. Evans $\cdot$ K. N. Barnett $\cdot$ S. A. Ogston

Division of Community Health Sciences,

University of Dundee Medical School,

Dundee, Scotland, UK

\section{A. D. Morris}

Division of Medicine and Therapeutics, University of Dundee Medical School,

Dundee, Scotland, UK

J. M. M. Evans $(\square)$

Section of Public Health,

Division of Community Health Sciences,

Mackenzie Building, Kirsty Semple Way,

Dundee DD2 4BF, Scotland, UK

e-mail: j.m.m.stansfield@dundee.ac.uk incidence and mortality had remained at 1993 levels, there would have been an increase in prevalence of 855 per 100,000 in 2003 , accounting for $60.1 \%$ of the actual increase of 1,423 per 100,000 . If there had been no mortality decrease, prevalence in 2003 would have been very similar to the actual prevalence observed.

Conclusions/interpretation Decreasing mortality rates in Tayside had less effect on the increase in prevalence than did increasing incidence. Even if incidence and mortality remain unchanged, prevalence will increase by over $20 \%$ in the next decade.

Keywords Diabetes mellitus · Incidence · Prevalence · Type 2 diabetes

Abbreviations
$\begin{array}{ll}\text { DARTS Diabetes Audit and Research in Tayside, } \\ \text { Scotland }\end{array}$

\section{Introduction}

The global increase in prevalence of type 2 diabetes mellitus is widely acknowledged [1] and confirmed by population-based prevalence studies in the UK [2], the USA [3] and Europe [4]. However, for this truly to represent an 'epidemic' of diabetes there must also be an increase in incidence.

Prospective, population-based studies on the changing incidence of type 2 diabetes over time are few and changes in diagnostic criteria make them difficult to interpret. Diagnosed diabetes rates can only ever be a proxy for the true incidence rate. Nevertheless, evidence is accumulating 
that we are seeing a widespread increase in incidence [5]. For example, an increase of $41 \%$ in self-reported diabetes over 7 years in the USA was noted (although the diagnoses were retrospective) [6]. In the UK, there was a $26 \%$ increase in number of cases of diabetes diagnosed in 208 general practices between 1994 and 1998 [7].

In contrast, a study among 470,000 people in Fyn, Denmark, [8] showed no significant increase in the incidence of type 2 diabetes over a 7 -year period (although an important limitation was that people with diet-treated diabetes were excluded). However, there was still a $4 \%$ increase in prevalence over the time period. The authors highlighted the possible effects of a decrease in mortality on increasing prevalence of type 2 diabetes, which in 2003 led one author [9] to ask whether the increase in prevalence of diabetes in other populations might also be the result of people being diagnosed and treated earlier, and living longer. The authors of the Fyn study have also shown theoretically the considerable effect of declining mortality on the prevalence rate in the Fyn population, where a projected annual increase in patients requiring prescriptions for diabetes of $5 \%$ could be driven simply by declining mortality with a stable incidence rate [10].

The Diabetes Audit and Research in Tayside, Scotland (DARTS) diabetes clinical information system [11] was set up in 1993 in Tayside, a region of Scotland, UK. We used this system to determine prospectively changes in prevalence, incidence and mortality of type 2 diabetes over a 12year period; we also examined the relative contributions of increasing incidence and declining mortality to changes in prevalence.

\section{Subjects and methods}

Tayside, Scotland, had a population of 387,908 people in 2004. The DARTS clinical information system contains detailed clinical information on every patient in Tayside diagnosed with type 1 or type 2 diabetes (regardless of treatment) from January 1993 [11]. Data collection for diagnosed patients has been standardised since 1995 and performed according to a national clinical dataset. It includes collation of several routine datasets and ongoing retrieval of information directly from medical records in primary care, hospitals, retinal eye screening and laboratory services, including validation by a dedicated team of research nurses. Patients with type 2 diabetes are defined as people over the age of 35 years who are diagnosed with diabetes or younger patients for whom there is no immediate requirement for insulin. The DARTS system can be linked to national mortality records by record-linkage and is widely used for epidemiological research.
Mid-year population estimates for Tayside Health Board were obtained from the General Register Office for Scotland. The numbers of new cases of type 2 diabetes for every year between 1993 and 2004 were determined and incidence rates calculated (expressed per 100,000 population without a diagnosis of diabetes). The exact mid-year prevalence rates were calculated for every year by counting the numbers of patients who were diagnosed before midyear and removing those who died before this date (although we were not able to adjust for patients who might have moved out of Tayside after diagnosis). These rates were expressed per 100,000 population. Mortality rates (expressed per 1,000 people with type 2 diabetes) were calculated as the number of deaths of patients with type 2 diabetes divided by mid-year prevalence (available up to 2003 only). We used a logistic regression model to determine whether there was a statistically significant increasing trend in prevalence of type 2 diabetes. We used Poisson regression models to evaluate trends in incidence and mortality. The models for incidence and prevalence were adjusted for age.

We then determined the extent to which the increase in prevalence between 1993 and 2003 was due to increasing incidence or decreasing mortality. We modelled the increase in prevalence that would have occurred each year according to three sets of assumptions:

Scenario 1 mortality remained at the 1993 level but incidence rates increased as observed.

Scenario 2 incidence remained at the 1993 level but mortality rates decreased as observed.

Scenario 3 mortality and incidence remained at the 1993 levels.

We took into account population aging over time by calculating rates within four age groups $(0-44,45-64,65-$ 84 and $\geq 85$ years) and using population estimates for all age groups for all years between 1993 and 2004. We then compared the prevalence rates obtained for the three scenarios with the actual prevalence rate observed in Tayside in 2003 (Fig. 1). Finally, we determined what the prevalence of type 2 diabetes would be in 2013, if incidence and mortality remained at 2003 levels (assuming no population increase).

\section{Results}

The prevalence of type 2 diabetes in Tayside increased from 1,492 per 100,000 in 1993 to 3,130 per 100,000 in 2004. This represents more than a doubling over 12 years. In a logistic regression model, the mean annual increase was $6.7 \%(95 \%$ CI $6.5-6.9 \%, p<0.01)$. Tayside is a relatively stable population, therefore any over-estimates as a result of 


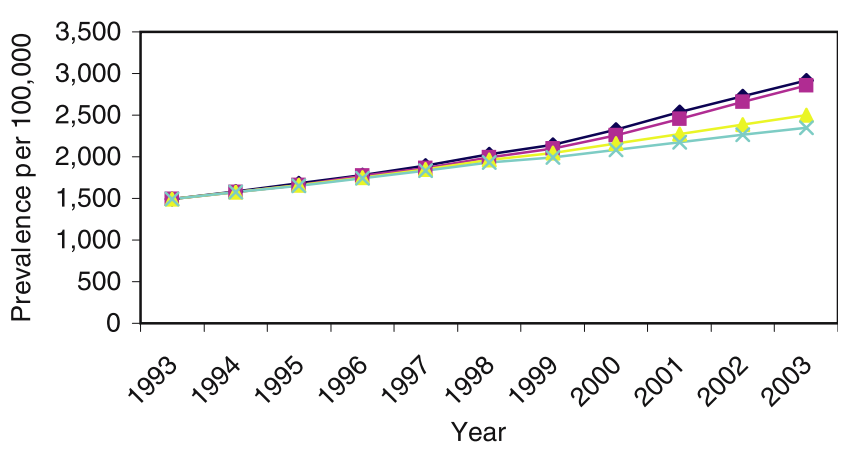

Fig. 1 Observed and modelled prevalence rates (per 100,000) of type 2 diabetes in Tayside. Scenario 1 (pink symbols): mortality at 1993 levels, incidence increasing as observed; Scenario 2 (yellow symbols): incidence at 1993 levels, mortality decreasing as observed; Scenario 3 (light blue symbols): mortality and incidence at 1993 levels. Dark blue symbols, observed prevalence rates

patients moving out of Tayside after diagnosis are likely to be small.

There was also a doubling of the incidence of type 2 diabetes in Tayside over the 12-year period. Using a Poisson model, the mean annual increase in incidence of type 2 diabetes, adjusted for age, was 6.3\% (95\% CI 5.7$6.9 \%, p<0.01)$. The mortality rate among people with type 2 diabetes declined from 69.0 per 1,000 diabetic patients in 1993 to 53.9 per 1,000 in 2003 (with a maximum value of 70.5 in 1995 and a minimum of 47.7 in 2002). Although this rate showed more variation, there was still a significant trend in a Poisson model, with a mean annual decrease in mortality of $3.7 \%(p<0.01)$.

The increase in prevalence of type 2 diabetes between 1993 and 2003 was 1,423 per 100,000 (from 1,492 to 2,915 per 100,000). Figure 1 and Table 1 demonstrate that if incidence and mortality had remained at the 1993 levels (Scenario 3), the increase in prevalence would have been 855 per 100,000 (to 2,347 per 100,000). This accounts for $60.1 \%$ of the actual increase observed. If incidence had remained the same, but mortality had decreased (Scenario 2), there would have been an additional increase of only 155 per 100,000 compared with Scenario 3, i.e. $10.9 \%$ of the actual increase. If mortality had remained the same, but incidence had increased (Scenario 1), there would have been a further increase of 355 per 100,000 compared with Scenario 2, i.e. $24.9 \%$ of the actual increase. The remaining increase in prevalence is the effect of increasing incidence and decreasing mortality occurring simultaneously.

The similarity in Fig. 1 between observed prevalence and prevalence in Scenario 1 (no mortality decrease) also highlights the small impact that mortality decrease had on prevalence in Tayside.

We calculated that if incidence and mortality remain at 2003 levels, by 2013 the prevalence of type 2 diabetes in Tayside will be 3,550 per 100,000 population (a $22 \%$ increase).

\section{Discussion}

This population-based study, using a validated clinical information system, clearly shows that there has been a doubling of prevalence and incidence of type 2 diabetes in a region of Scotland over 12 years, trends that are likely to be reflected in the UK. We are confident that we have identified real increases. While spurious effects might be the result of introducing the diabetes register in Tayside in 1993, it is unlikely that these effects would persist over a lengthy period. In 1999, the WHO lowered its criteria for diagnosis of diabetes by fasting plasma glucose values from $\geq 7.8$ to $7.0 \mathrm{mmol} / \mathrm{l}$, and this may have affected absolute incidence rates. However, the rate of increase in incidence is similar before and after 1999 (data not shown). All diagnoses are validated, and the register has higher sensitivity than general practice lists [11], perhaps explain-

Table 1 Observed and projected prevalences, incidence rates and mortality rates

\begin{tabular}{|c|c|c|c|c|c|c|}
\hline & $\begin{array}{l}\text { Observed mid-year } \\
\text { prevalence per } \\
100,000\end{array}$ & $\begin{array}{l}\text { Observed } \\
\text { incidence per } \\
100,000\end{array}$ & $\begin{array}{l}\text { Observed mortality rate } \\
\text { per } 1,000 \text { diabetic people }\end{array}$ & $\begin{array}{l}\text { Scenario 1: } \\
\text { prevalence per } \\
100,000\end{array}$ & $\begin{array}{l}\text { Scenario } 2 \text { : } \\
\text { prevalence per } \\
100,000\end{array}$ & $\begin{array}{l}\text { Scenario } 3 \text { : } \\
\text { prevalence per } \\
100,000\end{array}$ \\
\hline 1993 & $1,491.6$ & 187.5 & 69.0 & $1,491.7$ & $1,491.7$ & $1,491.7$ \\
\hline 1994 & $1,582.1$ & 191.1 & 66.7 & $1,574.2$ & $1,574.2$ & $1,574.2$ \\
\hline 1995 & $1,684.3$ & 210.0 & 70.5 & $1,657.5$ & $1,659.1$ & $1,654.4$ \\
\hline 1996 & $1,780.4$ & 194.3 & 63.2 & $1,771.4$ & $1,748.7$ & $1,746.1$ \\
\hline 1997 & 1,895 & 219.2 & 51.5 & $1,865.0$ & $1,846.4$ & $1,834.2$ \\
\hline 1998 & $2,031.6$ & 234.9 & 56.8 & $1,990.7$ & $1,965.7$ & $1,928.3$ \\
\hline 1999 & $2,146.3$ & 258.4 & 54.7 & $2,100.3$ & $2,045.8$ & $1,993.6$ \\
\hline 2000 & $2,322.3$ & 292.6 & 49.2 & $2,259.4$ & $2,157.2$ & $2,087.0$ \\
\hline 2001 & $2,534.8$ & 322.3 & 48.1 & $2,456.1$ & $2,273.5$ & $2,176.3$ \\
\hline 2002 & $2,729.9$ & 328.0 & 47.7 & $2,659.4$ & $2,389.6$ & $2,263.4$ \\
\hline 2003 & $2,915.3$ & 344.5 & 53.9 & $2,856.7$ & $2,502.4$ & $2,347.1$ \\
\hline 2004 & $3,129.9$ & 375.2 & - & - & - & - \\
\hline
\end{tabular}


ing why we identified an even higher increase in prevalence than a previous UK-based study over a similar time period [2].

Our simple models emphasise two very important points. We have shown that more than half of the increase in prevalence between 1993 and 2003 would have occurred anyway, even if incidence and mortality had remained unchanged from 1993 levels. This is because the number of patients being diagnosed with type 2 diabetes every year is greater than the number of type 2 diabetic patients who die. Even if incidence does not increase from 2003 levels (an extremely unlikely scenario), we can still expect more than a 20\% increase in prevalence by 2013 (assuming stable mortality rates). Note also that this figure does not take into account the increasing proportions of old people in the population by 2013 and is therefore almost certainly an underestimate.

We have also shown that in Tayside, Scotland, increasing incidence rates had three times the effect on the prevalence of type 2 diabetes between 1993 and 2003 than did decreasing mortality rates.

Acknowledgements We thank the members of the DARTS Steering Group for their support. We also thank the support staff of the DARTS/Medicines Monitoring Unit (MEMO) Collaboration who facilitated this work. This work was part of a larger study funded by the Chief Scientist Office, Scotland.

Duality of interest The authors had no conflicts of interest with this paper.

\section{References}

1. King H, Aubert RE, Herman WH (1998) Global burden of diabetes, 1995-2025. Prevalence, numerical estimates and projections. Diabetes Care 21:1414-1431

2. Fleming DM, Cross KW, Barley MA (2005) Recent changes in the prevalence of diseases presenting for health care. Br J Gen Pract 55:589-595

3. Leibson CL, O'Brien PC, Atkinson E, Palumbo PJ, Melton LJ 3rd (1997) Relative contributions of incidence and survival to increasing prevalence of adult-onset diabetes mellitus: a population-based study. Am J Epidemiol 146:12-22

4. Passa P (2002) Diabetes trends in Europe. Diabetes Metab/Res Rev 18:S3-S8

5. Burke JP, Williams K, Gaskill SP, Hazuda HP, Haffner SM, Stern MP (1999) Rapid rise in the incidence of type 2 diabetes from 1987 to 1996: results from the San Antonio Heart Study. Arch Int Med 159:1450-1456

6. Geiss LS, Pan L, Cadwell B, Gregg EW, Benjamin SM, Engelgau MM (2006) Changes in incidence of diabetes in US adults 1997 2003. Am J Prev Med 30:371-377

7. Ryan R, Newnham A, Khunti K, Majeed A (2005) New cases of diabetes mellitus in England and Wales, 1994-1998: database study. Public Health 119:892-899

8. Stovring H, Andersen M, Beck-Nielsen H, Green A, Vach W (2003) Rising prevalence of diabetes: evidence from a Danish pharmaco-epidemiologic database. Lancet 362:537-538

9. Gale EAM (2003) Is there really an epidemic of type 2 diabetes? Lancet 362:503-504

10. Green A, Stovring H, Andersen M, Beck-Nielsen H (2005) The epidemic of type 2 diabetes is a statistical artefact. Diabetologia 48:1456-1458

11. Morris AD, Boyle DIR, MacAlpine R et al (1997) The diabetes audit and research in Tayside Scotland (DARTS) study: electronic record-linkage to create a diabetes register. BMJ 315:524-528 